\title{
No linealidad en muestras cerámicas de PZT muy delgadas
}

\author{
J.DE FRUTOS ${ }^{1}$, A.M.GONZÁLEZ ${ }^{1}$, M.C. DURO' ${ }^{1}$, J.F. FERNÁNDEZ² \\ ${ }^{1}$ E.T.S.I.Telecomunicación. Ciudad Universitaria s/n. 28040 Madrid \\ ${ }^{2}$ Dpto. Electrocerámica, Instituto de Cerámica y Vidrio, CSIC. Cra Valencia km 23,4. Arganda del Rey (Madrid)
}

\begin{abstract}
El desarrollo de sensores piezoeléctricos de alta frecuencia, exige trabajar con muestras de material muy delgadas, del orden de micras. En estos espesores, las tensiones de trabajo causan fuertes perturbaciones en los materiales, provocando respuestas no lineales, sumamente dependientes de las condiciones de trabajo. Con el objetivo de avanzar en el conocimiento de este fenómeno, y haciendo uso de la técnica de Espectroscopia de Impedancia Compleja, hemos analizado la respuesta de muestras de PZT cerámico adelgazadas a espesores de micras, en función del campo aplicado y de la amplitud de la señal de análisis. El estudio de estos resultados, puede permitir sentar las bases para el desarrollo de sensores ultrasónicos de alta frecuencia sintonizables.
\end{abstract}

Palabras clave: Piezoelectricidad, resonancia, sensores, no linealidad.

\section{No lineal response in thin samples OF PZT ceramics.}

The development of piezoelectric sensors for high frequency requires to work with very thin samples of material. At this thick nesses, the stress concentration produces strong perturbations in the materials, and the origin oF a non linear response, which depends field on working conditions. With the goal to advance in the knowledgement of this phenomenon, and by using Complex Impedance Spectroscopy, we have analysed the response of PZT ceramics with thickness of microns as a function of the applied and the amplitude of the analysis signal. These results allow to establish the base to the development of ultrasonic tunable sensors for high frequency.

Keywords: piezoelectricity, resonance, sensors, non linear response

\section{INTRODUCCIÓN.}

Desde los años 60 se vienen utilizando los materiales ferroeléctricos como elementos básicos en medicina para la monitorización de órganos o fetos, Sistemas Ecográficos, o para el tratamiento de cólicos renales, y la destrucción de piedras en el riñón, haciendo uso de dispositivos ultrasónicos. Con el desarrollo de nuevos materiales, y la mejora de sus propiedades, los niveles de monitorización alcanzados, tanto en sistemas ecográficos como termográficos, son altísimos. Permitiendo en la actualidad el seguimiento de arritmias cardiacas, la determinación del flujo sanguíneo, la detección de tejidos defectuosos, cáncer de colon y un sinfín de patologías, imposible de ser detectada por otras técnicas, y en todo caso, sin los efectos secundarios asociados a Rayos X o sistemas de análisis basados en radiación con ondas electromagnéticas. Desde los años setenta, se utilizan además dispositivos y aplicaciones basadas en estos materiales para el tratamiento de diferentes patologías, fundamentalmente en procesos de rehabilitación, en cirugía y en odontología (1-6). Como muestra, podemos referirnos a las sondas rectales de análisis, detección y microcirugía ultrasónica de cáncer de próstata $(7,8)$, aunque el número de aplicaciones en este campo aumentan día a día. Las tendencias actuales en este campo, tienden por una parte al desarrollo de actuadores de frecuencias resonantes mayores, fundamentalmente en el rango de 30 a $100 \mathrm{MHz}$, y a aumentar la potencia emisora de los mismos (6,9-11). El mayor rango de frecuencia favorece la capacidad de información de la muestra al obtener una mejor penetración de la señal dentro del material, y conseguir mayores niveles de potencia que favorecen el poder utilizar el mismo elemento como dispositivo de análisis y como dispositivo quirúrgico. El tratar de combinar ambos procesos implica por una parte reducir el espesor de los elementos pulsadores al rango de micras, y por otra, trabajar con tensiones de trabajo relativamente grandes provocando comportamientos no lineales en el material (9-14). En este trabajo hacemos un estudio de este comportamiento no lineal y proponemos nuevas aplicaciones para este tipo de dispositivos.

\section{EXPERIMENTAL}

Se ha trabajado con muestras de PZT comercial, montado en avisadores acústicos. En estos dispositivos el elemento cerámico se encuentra pegado a una chapa metálica de $0.1 \mathrm{~mm}$ de espesor, y el propio material tiene un espesor de $0.1 \mathrm{~mm}$. Para disponer de muestras de espesor diversos, se procede a un pulido en cuña del material como se indica en la figura 1 . De esta forma se dispone de gama de muestras de las mismas características, pero de diferentes espesores, todos ellos en el rango de 1 a $20 \mu \mathrm{m}$.

Inicialmente el material estaba polarizado, pero tras el proceso de pulido y posterior tratamiento térmico para relajar las tensiones inducidas, se puede asumir que está despolarizado.

El estudio del material, se ha realizado basándose en dos aspectos diferentes: 
Respuesta del material despolarizado y polarizado ante diferentes amplitudes de la tensión de caracterización.

Seleccionando una amplitud de análisis relativamente pequeña para que no perturbe en exceso las medidas, respuesta del material con una tensión continua aplicada, en la misma dirección, y en la opuesta a la del campo polarizante aplicado con anterioridad.

Los estudios se han realizado por medio de los puentes de impedancia HP-4291A y HP-4192. Esto nos permite disponer de barridos en frecuencia de $100 \mathrm{~Hz}$ a $1.8 \mathrm{GHz}$, y en el rango de hasta $40 \mathrm{MHz}$ podemos además determinar el comportamiento de las muestras bajo la acción de una tensión continua de hasta $200 \mathrm{~V}$, que para los espesores de nuestras muestras permite campos aplicados con valores del orden de $\mathrm{MV} / \mathrm{cm}$

\section{RESULTADOS Y DISCUSIÓN}

\subsection{Dependencia con la amplitud del campo oscilante aplicado.}

Las primeras medidas que se hicieron corresponden a medidas en las que se variaba la amplitud de la tensión oscilante de análisis desde $10 \mathrm{mV}$ a $1 \mathrm{~V}$. Previamente, las muestras fueron polarizadas a temperatura ambiente con un campo continuo de $70 \mathrm{kV} / \mathrm{cm}$.

Se analizó en todo caso, la presencia de modos resonantes en las muestras $(15,16)$, y fundamentalmente las posibles desviaciones de las frecuencias de los mismos al someterles a campos oscilantes, siempre menores que el de polarización. En la figura 2 puede verse el resultado para una resonancia. Tanto en esta figura como en el resto de resultados observados, se comprueba que existe una ligera variación en la respuesta de las muestras pero que en todo momento es poco significativa.

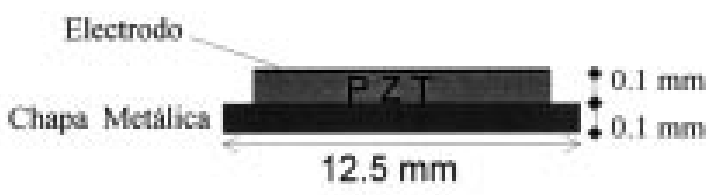

\section{MUESTRA DE PARTIDA}

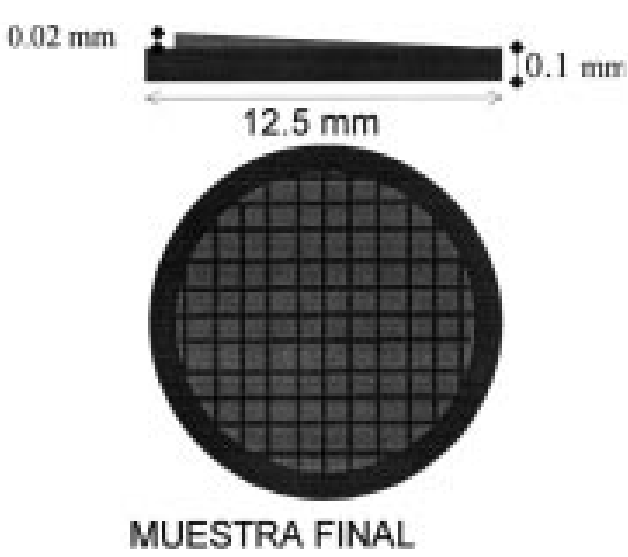

Fig. 1. Proceso seguido en la preparación de muestras.

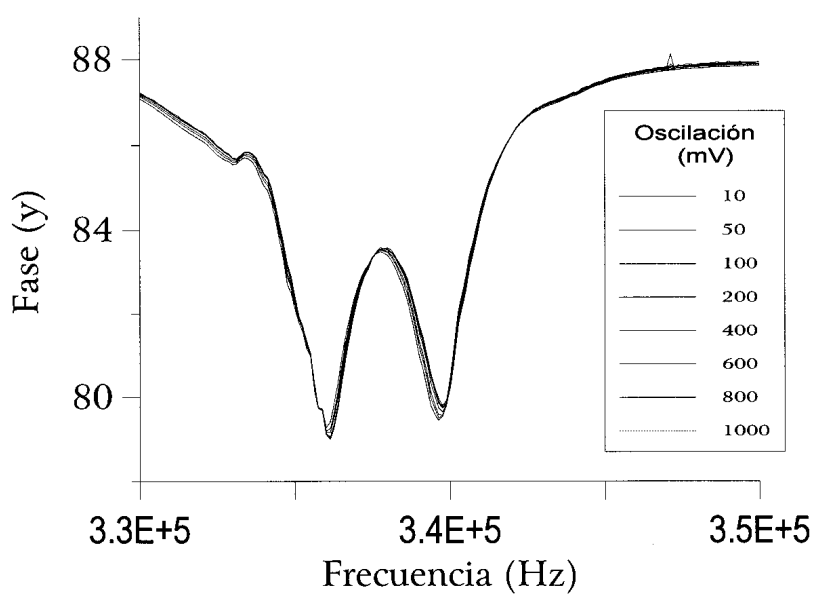

Fig. 2. Respuesta dieléctrica de una muestra de 20 micras, en función de la frecuencia, para diferentes campos de análisis.

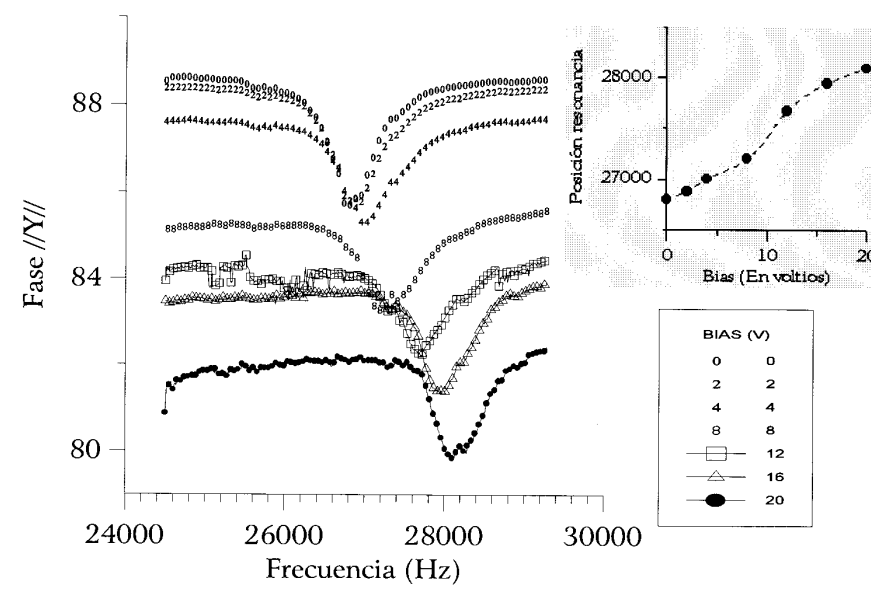

Fig. 3 Respuesta en función del Bias aplicado. La dirección del Bias coincide con la del campo de polarización

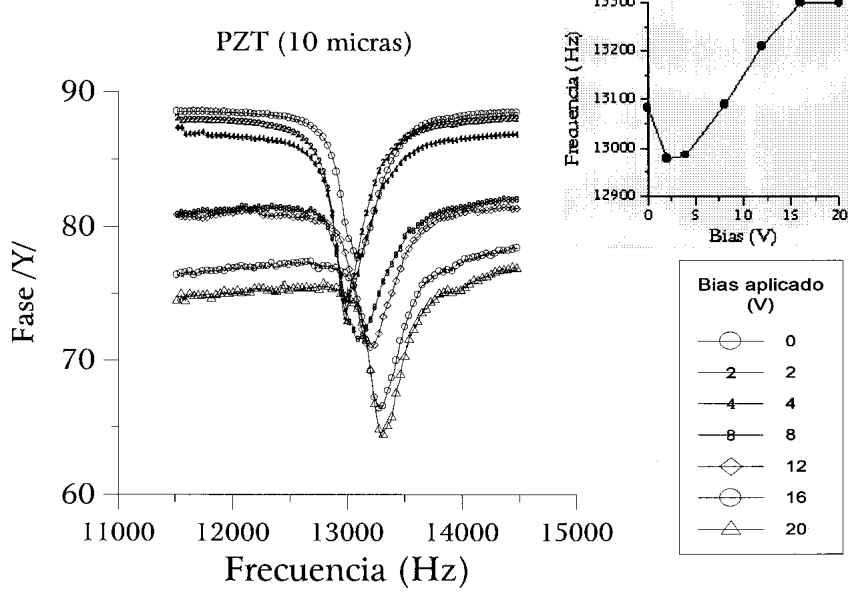

Fig. 4 Respuesta en función del Bias aplicado. La dirección del Bias es opuesta a la del campo de polarización 


\subsection{Dependencia con la tensión "Bias" aplicada}

El estudio con el "Bias" aplicado (17-19), se realiza en muestras de diferentes espesores, en el rango decenas de micras, obteniéndose resultados similares para los diferentes espesores estudiados. Con bajos niveles de campo aplicado, se adquiere un nivel de polarización en las muestras proporcionando numerosas frecuencias de resonancia. Debido a lo delgado de la muestra, a lo irregular de la misma y especialmente a estar unida al soporte metálico es difícil relacionar las diferentes resonancias con modos propios de las muestras. No obstante, a frecuencias altas se aprecian modos resonantes que se pueden asociar a resonancias en espesor. En muestras de 10 micras se han observados modos resonantes en alta frecuencia, con un modo fundamental en aproximadamente 340 $\mathrm{MHz}$, y un primer armónico en aproximadamente 1,03 GHz. Esta relación, y las características y espesor de la muestra, permiten asumir que se trata de modos resonantes en espesor.

Las figuras 3 y 4 muestran alguno de los resultados más importantes obtenidos en estas condiciones de trabajo. La Figura 3 corresponde a los resultados obtenidos en una muestra previamente polarizada a temperatura ambiente, con un campo aplicado de 70 Voltios, aproximadamente $70 \mathrm{KV} / \mathrm{cm}$. Se ha caracterizado la respuesta de la muestra, mientras se aplican diferentes campos continuos, con la misma orientación que el utilizado para polarizar la muestra. Puede observarse un marcado desplazamiento de la posición de los máximos de resonancia con el Campo aplicado, que como puede comprobarse en el detalle superior de la figura, presenta un desplazamiento prácticamente lineal y en medidas sucesivas sobre la misma muestra, se ha comprobado que el resultado es reproducible, siempre que no se altere la dirección del campo aplicado respecto al utilizado para polarizar la muestra.

Por su parte, la figura 4 muestra los resultados obtenidos cuando el campo aplicado en la caracterización de la muestra es opuesto al utilizado en el proceso de polarización. El resultado más llamativo, es el cambio que se observa en el desplazamiento de los máximos relativos. Parece desprenderse de estos resultados que la muestra invierte su polarización con campos relativamente pequeños, y una vez que se produce este hecho, mantiene un desplazamiento de las frecuencias resonantes similares a los obtenidos cuando el campo continuo mantiene la misma dirección del campo utilizado previamente para polarizar las muestras.

Los desplazamientos de los máximos de resonancia, pueden justificarse en base a las tensiones inducidas en las muestras por los campos aplicados. Estas tensiones realizan la misma función que las cargas de masa para el ajuste fino de frecuencias resonantes en los osciladores comerciales de cuarzo. La ventaja fundamental de nuestro caso, es que los procesos son reversibles, y fácilmente controlables.

\section{CONCLUSIONES}

Se han mecanizado muestras de PZT comercial, consiguiendo muestras de espesores que varían entre micras y centenas de micras.

Se ha estudiado la respuesta de las mismas en función de la frecuencia, para diferentes amplitudes del campo oscilante de caracterización, y para diferentes campos continuos aplicados durante el proceso de caracterización. Se pone de manifiesto, que mientras las variaciones de la amplitud de oscilación prácticamente no influyen en la respuesta de las muestras, cuando se varía la intensidad del campo continuo aplicado se obtiene una fuerte dependencia de las posiciones de los máximos de resonancia con este parámetro.

Los estudios presentados de dependencia de la posición de los máximos de resonancia con el campo continuo aplicado, su comportamiento cuando mantienen la misma dirección del campo de polarización, junto con la facilidad para invertir la dirección de polarización con campos reales pequeños, permiten afirmar que este tipo de muestras pueden utilizarse como sensores piezoeléctricos sintonizables.

El desplazamiento de las posiciones de los máximos de resonancia con los campos, es debido a las tensiones inducidas en el material por el campo continuo aplicado. De esta forma, la sintonización puede controlarse de forma simple variando la tensión.

\section{AGRADECIMIENTOS}

Este trabajo ha sido financiado por la CICYT española, por medio del proyecto de investigación MAT97-0694-C02

\section{BIBLIOGRAFÍA}

1. T.R. Gururaja. "Piezoelectrics for medical ultrasonic imaging". Am. Ceram. Soc. Bull, Vol 73, 50-57, 1994

2. F. S. Foster. Medical and biological imaging with frequency ultrasound, IEEE Trans. Ultras. Ferr. Freq. Cont., Vol 42, 485-491 (1995).

3. A. Safari. "Novel piezoelectric ceramics and composites for sensor and actuator applications". Mat. Res. Innovat. 2, 263-269, 1999.

4. W. A. Grandia, C. M. Fortunko. "NDT Applications for Air-Coupled Ultrasonic Transducers". IEEE Ultrasonics Symposium 1, 697-709(1995).

5. G.R. Lockwood, D.H. Turnbull, D.A.Christopher, And F.S. Foster. "Beyond 30 $\mathrm{MHz}$ - applications of high frequency ultrasound imaging". IEEE Engineering In Medicine And Biology, 15, 60-71(1996)

6. F. S. Foster. "Characterization of lead zirconate titanate ceramics for use in high- frequency $(20-80 \mathrm{MHz})$ transducers". IEEE Trans. Ultras. Ferr. Freq. Cont., 38, 446-453(1994).

7. A. Fenster, S. Tong, H.N. Cardinal, C. Blake, D.B. Downey. "Three dimensional ultrasound imaging system for Prostate Cancer Diagnosis and Treatment". IEEE Inst. Meas. Mag. 12, 32-35 (1998)

8. A. Fenster, D.B. Downey. 3D-ultrasound imaging: A review. “IEEE Eng. Med. and Biology" . 15, 41-51 (1996)

9. M.L.Calzada, J. de Frutos. "Chemical processing and microstructure of piezoelectric lead titanate ceramics". J. Mat Sci.: Mat. Electronics. 4, .83-88, (1993)

10. B.Jiménez, J. de Frutos "Piezoelectric relaxation studies of ferro-piezoelectric ceramics Ferroelectrics", 109, 107-112. (1990)

11. A.M. González, J.de Frutos, M.C. Duro, N. Duang, N. Cereceda, J.A. Gonzalo. "Estudio de propiedades de materiales de PZT en función de la composición en la región de la frontera morfotrópica" Bol. Soc. Esp. Cerám. Vidrio, 37 [2-3], 172-175 (1998)

12. T.E. Gómez Alvarez-Arenas, A.M.González, J. de Frutos, F.R. Montero de Espinosa. "Piezoelectric characterization of porous piezoceramics". 1996 IEEE Ultr. Sound Sym. 519-523 (1996)

13. S.E. Park And T.R. Shrout. "Characteristics of relaxor based piezoelectric single cystals for ultrasonic transducer". IEEE Trans. Ultrason. Ferro. Freq. Control 44, 1140-1147 (1997)

14. A.M.González, J. de Frutos, M C. Duro, C.Alemany, L. Pardo. "Changes in the piezoelectric of PZT ceramics during the poling process". Ferroelectrics, 208,. 449-457 (1998)

15. R. Perez, E. Minguella, J.A. Gorri "Weak Nonlinearities in piezoelectric transducers. Equivalent circuits". Proceeding Of ISAF 98, IEEE 247-250 (1999) 16. D. Guyomar, N. Aurelle, C. Richard, P. Gonnar, L.Eyraud. Nonlinearities In Langevin Transducers "IEEE Proc. Ultrasonics International", 2, 925-928 (1994)

17.A.M.González, J. de Frutos, C. Alemany, L.Pardo. “Evolution of dielectric constant of a piezoelectric ceramics during poling". Proceedings Of ELECTROCERAMICS V,. Editado Por: Fundacâo Joâo Jacinto De Magalhàes. Aveiro. Portugal 143-147 (1996)

18. A.M.González, J. de Frutos, C.Alemany Y L. Pardo. "Variation of the piezoelectric complex parameters of thin ceramic disks during poling". Proc. ISAF'96 Series IEEE; 96CH35948 (1997)

19. V. Mueller, Q.M. Zhang. "Nonlinearity and scaling behaviour in donor-doped Lead Zirconate Titanate Piezoceramics". Appl. Phys.Lett., Vol 72, 2692-2694 (1998) 